\title{
Experimental Investigation of the Parallel Vortex-Airfoil Interaction at Transonic Speeds
}

\author{
Iraj M. Kalkhoran* \\ Polytechnic University, Farmingdale, New York 11735 \\ and \\ Donald R. Wilson $\dagger$ \\ University of Texas at Arlington, Arlington, Texas 76019
}

\begin{abstract}
Unsteady vortex-airfoil interaction experiments at transonic Mach numbers ranging from 0.7 to 0.85 and airfoil chord Reynolds numbers of $3.5 \times 10^{6}$ to $5.5 \times 10^{6}$ were conducted in the University of Texas at Arlington high Reynolds number transonic wind-tunnel facility. The experiments were designed to simulate a two-dimensional blade-vortex interaction problem frequently encountered in rotocraft applications. The interaction experiments involved positioning a two-dimensional vortex generator upstream of a NACA 0012 airfoil section and impulsively pitching the vortex generator airfoil such that the starting vortex interacts with the downstream airfoil. The vortex generator was pitched about its 0.25 chord position with nondimensional pitch rates in the range of 0.004-0.008. Experiments were conducted at several vortex core positions above and below the downstream airfoil. The results indicate a substantial change in the pressure distribution over the leading $30 \%$ of the interacting airfoil. Experimental data for Mach numbers representing supercritical flows over the airfoil resulted in a very strong interaction of the vortex and the airfoil shock wave; at close encounters, these interactions resulted in unsteady local flow separation of the leading $40 \%$ of the airfoil chord. Experiments with stronger vortices at supercritical Mach numbers resulted in a forward propagation of the shock wave. The pressure distribution of the downstream airfoil was not sensitive to Reynolds number variations for the range of Reynolds numbers simulated in the test program.
\end{abstract}

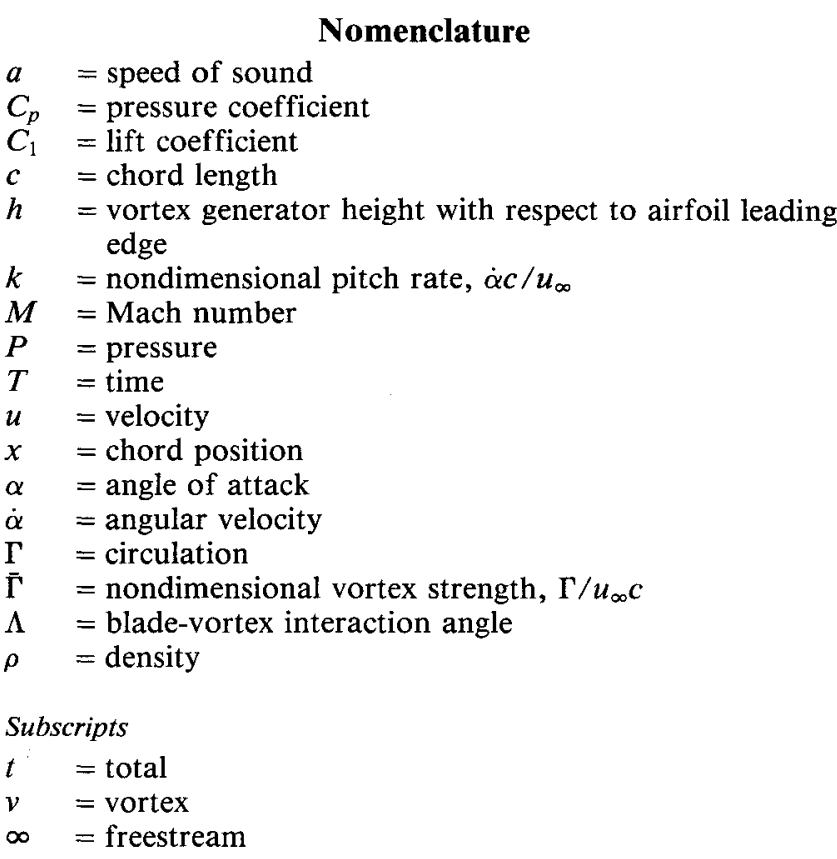

\section{Introduction}

$\mathbf{T}$

HE interaction of the tip vortex shed from one blade with the other blades of a helicopter rotor is known to have a marked effect on both the aerodynamic and acoustic charac-

Presented as Paper 89-1833 at the AIAA 20th Fluid Dynamics, Plasma Dynamics, and Lasers Conference, Buffalo, NY, June 12-14, 1989; received May 15, 1991; revision received Jan. 22, 1992; accepted for publication Jan. 30, 1992. Copyright (C) 1992 by the American Institute of Aeronautics and Astronautics, Inc. All rights reserved.

*Assistant Professor, Department of Aerospace Engineering. Member AlAA.

$\dagger$ Professor, Aerospace Engineering Department. Associate Fellow AIAA. teristics of the helicopter. The blade-vortex interaction (BVI) has been identified as the source of unsteady aerodynamic loading of the blades, as well as the acoustic phenomenon known as blade slap. ${ }^{1}$ The BVI is most likely to occur during a powered descent and generally involves a three-dimensional unsteady interaction of blades with vortices shed from the same or the following blades. In order to enhance the performance level of future helicopters, understanding the BVI phe-
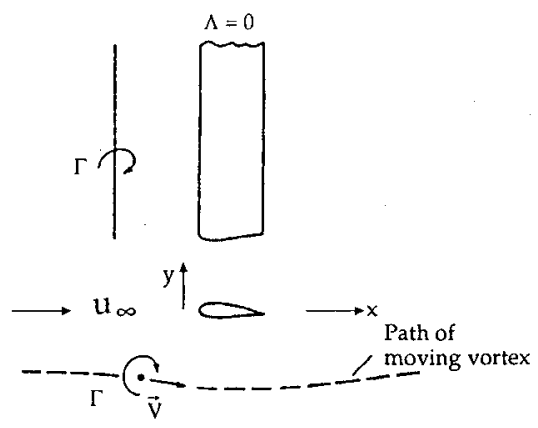

a) Vortex parallel to leading edge

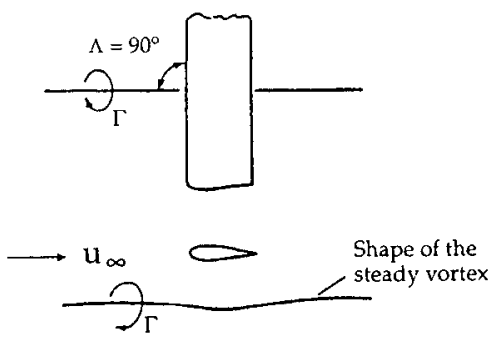

b) Vortex parallel to freestream

Fig. 1 Parallel and perpendicular blade-vortex interactions (from Ref. 15). 
nomenon is believed to be of vital importance both from environmental concerns and also military detectability considerations. Theoretical and experimental investigations of the BVI problem have most often concentrated on interaction of a vortex with a stationary airfoil or the so-called vortex-airfoil interaction. The limiting cases for such interactions are represented by the parallel and perpendicular interactions (Fig. 1). Such idealization has resulted in treating the parallel BVI as a two-dimensional, unsteady problem, whereas the perpendicular BVI is considered a steady but highly three-dimensional flow problem.

Because of the presence of shock waves and local supersonic regions over helicopter blades operating at transonic tip Mach numbers, the most pronounced BVI effects are known to occur at transonic blade speeds. However, most of the previous studies have concentrated on the aeroacoustics of the BVI problem at relatively low speeds. ${ }^{2-7}$

The low-speed aerodynamic aspects of the parallel BVI were reported by Seath et al. ${ }^{6}$ and Straus et al. ${ }^{7}$ Their scheme involved placing a vortex generator upstream of a two-dimensional airfoil and impulsively pitching the vortex generator such that its starting vortex interacted with the downstream airfoil. Their results indicated a significant alteration of the pressure distribution during the interaction, with the most pronounced effects concentrated near the leading edge of the airfoil. Caradonna et al. $^{8}$ investigated the parallel BVI by mounting an instrumented rotating blade downstream of a vortex generator in a low-speed wind tunnel. The blade rpm was set to simulate the parallel BVI at transonic relative Mach numbers. Two propagation mechanisms were observed in their experiments: a type $\mathrm{C}$ shock propagation at high tip speeds, and a rapid but continuous pressure pulse associated with the proximity of the vortex at lower tip speeds. Booth, ${ }^{9}$ Booth and $\mathrm{Yu},{ }^{10}$ and Poling et al. ${ }^{11}$ modeled the parallel BVI encounter by placing an airfoil downstream of an oscillating airfoil such that discrete vortices generated by the vortex generator interacted with the downstream airfoil. The drawback of such a scheme is believed to be due to the closeness of wake vortices to each other so that effects of individual vortices are not detectable. Booth ${ }^{9}$ used hot-wire measurements in the wake of the oscillating airfoil to determine the strength of vortices and performed limited surface pressure measurements during the unsteady encounter. The use of long tubing from the pressure taps to the transducers in the study reported by Booth makes the results questionable for time-accurate analysis of the data. Another technique for simulating the parallel BVI is reported by Meier and Timm $^{12}$ and Mandella and Bershader, ${ }^{13}$ in which the vortices are generated by impulsive starting of the flow over an airfoil mounted at an angle of attack in a shock tube. In their experiments, flow visualization via high-speed interferometry as well as sidewall pressure measurements were used to quantify the vortex intensity and the details of the interaction. Their results indicated a substantial transient disturbance to both lift and drag of the downstream airfoil.

On the computational side, several contributions have dealt with the transonic BVI problem. Srinivasan et al. ${ }^{14}$ used thinlayer Navier-Stokes, Euler, and transonic small disturbance equations to calculate the two-dimensional interaction of a vortex with an airfoil. They reported similar results for all

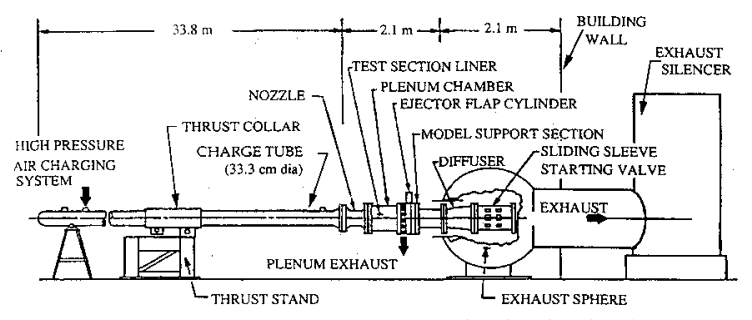

Fig. 2 Transonic Ludwieg-tube wind tunnel.

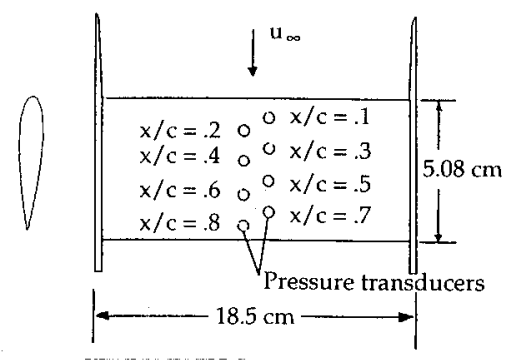

Fig. 3 Downstream airfoil geometry and transducer locations.

three schemes for weak interactions; whereas for stronger interactions, slight differences between the Euler and thinlayer Navier-Stokes solutions were observed. They, however, report a significant influence of the vortex on the flowfield around the airfoil. Furthermore, the vortex influences the pressure distribution over the entire airfoil chord. More recently, Srinivasan and McCroskey ${ }^{15}$ modeled the unsteady, three-dimensional flowfield of a helicopter rotor blade encountering a vortex. They used a finite difference numerical scheme with both Euler and thin-layer Navier-Stokes equations and reported good agreement with the experimental observations for both parallel and oblique interactions at subsonic and transonic blade tip speeds.

The present investigation was designed to measure the unsteady surface pressure distribution on an airfoil during a parallel vortex-airfoil interaction. The vortex was generated by impulsively pitching a full-span NACA 0012 airfoil section to shed a starting vortex. This vortex was then allowed to convect downstream and interact with a second instrumented airfoil. The experiments were conducted in the University of Texas at Arlington (UTA) high Reynolds number, Ludwiegtube wind tunnel ${ }^{16}$ at Mach numbers and Reynolds numbers representative of actual helicopter flight operations.

\section{Experimental Setup}

A line drawing of the transonic Ludwieg-tube wind tunnel is shown in Fig. 2. The tunnel has an $18.5 \times 23.3 \mathrm{~cm}(7.34 \times 9.16$ in.) rectangular porous wall test section and is capable of producing low-turbulence transonic flows at Mach numbers of $0.5-1.2$, and unit Reynolds numbers up to $4 \times 10^{8} / \mathrm{m}\left(10^{6} / \mathrm{in}\right.$.). The useful steady-state run time in the tunnel is $120-140 \mathrm{~ms}$.

The vortex generator consisted of a NACA 0012 airfoil section having a $5.08-\mathrm{cm}$ ( 2 -in.) chord that was mounted to a pitch actuation mechanism located at the interface between the nozzle and test section. The vortex generator airfoil had a span of $18.5 \mathrm{~cm}$ (7.34 in.) and was mounted vertically at the tunnel centerline, thus spanning the entire height of the test section. The vortex generator is impulsively pitched during the steady state portion of the wind tunnel run by means of a high-speed Tomkins-Johnson model SH-2 pneumatic actuator. A potentiometer was attached to the actuator in order to measure the actual pitch angle time history of the vortex generator. Maximum pitch rates of about $35 \mathrm{rad} / \mathrm{s}$ could be achieved with this system, which corresponds to a nondimensional pitch rate $\dot{\alpha} c / u_{\infty}$ of the order of 0.008 .

An instrumented NACA 0012 airfoil section with a $5.08-\mathrm{cm}$ (2-in.) chord length, also spanning the entire height of the test section, was mounted vertically $43.18 \mathrm{~cm}$ (17 in.) downstream of the vortex generator airfoil. The model has approximately $3.2 \%$ blockage in the test section. The airfoil was equipped with eight fast response surface mounted Kulite LQ-080-100 pressure transducers. The pressure transducers were located along the airfoil chord at every $0.1 c$, with the first transducer at the $10 \%$ chord station and the last transducer at the $80 \%$ chord station. To minimize the wall interference effects, the pressure transducers were located at the airfoil midspan location and the airfoil was instrumented on one side only (Fig. 3). The end plates of the downstream interacting airfoil were designed such that it could be positioned at various horizontal 


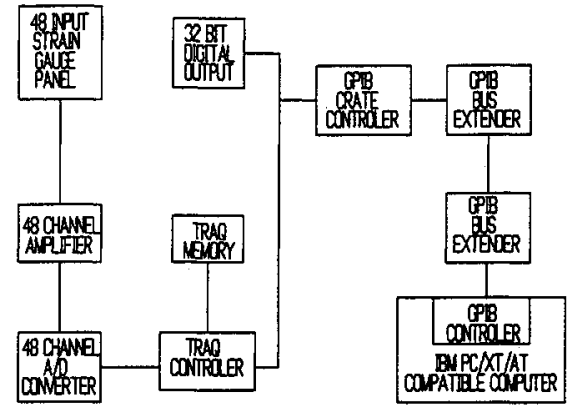

Fig. 4 Data acquisition and control system.

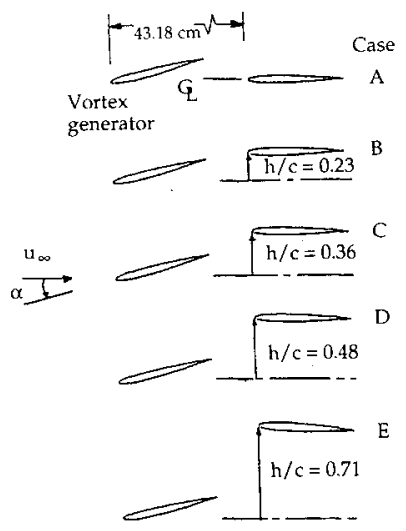

Fig. 5 Comparison of measured upper surface pressure distribution with the data of Ref. 18 for a nominal Mach number of 0.72 .

locations in the test section. Since the vortex generator was always located at the tunnel centerline, by positioning the downstream airfoil at various locations off test section centerline, vortex airfoil interactions with different incident separation distances could be achieved.

Facility operation, control, and data acquisition were accomplished by a DSP Technology data acquisition and control system (Fig. 4). The DSP Technology system provides 48 data channels, each having a dedicated variable gain amplifier, 12 bit $\mathrm{A} / \mathrm{D}$ converter, and a high-speed mass storage memory for storage of the digitized signals during a test run.

\section{Calibration Experiments}

The wind-tunnel calibration procedure consisted of simultaneous measurements of the charge tube stagnation pressure with total pressure probes located at the downstream end of the charge tube, the test section static pressure distribution with a standard centerline pipe, and the test section plenum cavity pressure with a plenum cavity rake. Details of the procedure are given in Ref. 17. The axial variation of the test section Mach number is about $0.5 \%$ for the range of Mach numbers tested. The results of a conventional uncertainty analysis were reported in detail in Ref. 17. For the range of data reported in this paper, the typical values of the uncertainty in the principle test variables are $M_{\infty}=0.015-0.018$, $R e=1.3-1.6 \times 10^{5}$, and $C_{p}=0.03-0.06$.

The airfoil calibration experiments were conducted for Mach numbers ranging from 0.7 to 0.85 , and Reynolds numbers of $3.5 \times 10^{6}$ to $5.5 \times 10^{6}$ based on the airfoil chord. The specific objectives of the airfoil calibration experiments were to verify the accuracy and repeatability of the airfoil pressure distribution and also to obtain a set of baseline data to be compared to the pressure distribution data during the unsteady vortex airfoil interaction experiments.

A difficulty encountered during the airfoil calibration studies was the deviation of the baseline pressure distribution from published experimental data for the NACA 0012 airfoil sec- tion. The reason for this discrepancy is believed to be a result of slight surface irregularities caused by the surface mounted pressure transducers. Figure 5 shows the steady-state surface pressure distribution without the vortex generator airfoil in place for the case of $M=0.72$. Deviation of the baseline pressure distribution from the published data was more pronounced for Mach numbers representing supercritical flows over the airfoil, as will be discussed later. Considering the sensitivity of the airfoil pressure distribution to small surface irregularities at transonic Mach numbers, only the vortex effects on the pressure distribution of the existing model will be presented, and the discussion will focus on the deviation of measured surface pressures from the baseline values.

\section{Experimental Results}

The vortex airfoil interaction tests were conducted at five incident vortex generator-airfoil vertical separation distances, as shown in Fig. 6. As already mentioned, to minimize test section wall interference, the airfoil surface pressure measurements were made on the lower surface only (closer to the test section centerline). The vortex generator was pitched counterclockwise about the quarter chord point, thus leading to trajectories of the shed starting vortex that include both passage over the upper surface (cases A and B) and below the lower surface (cases $C, D$, and $E$ ). The maximum pitch angle for these cases was equal to $+5 \mathrm{deg}$, which corresponds to an estimated nondimensional vortex strength of

$$
\bar{\Gamma}_{V}=\frac{\Gamma_{V}}{u_{\infty} c}=\frac{1}{2} C_{1}=0.25
$$

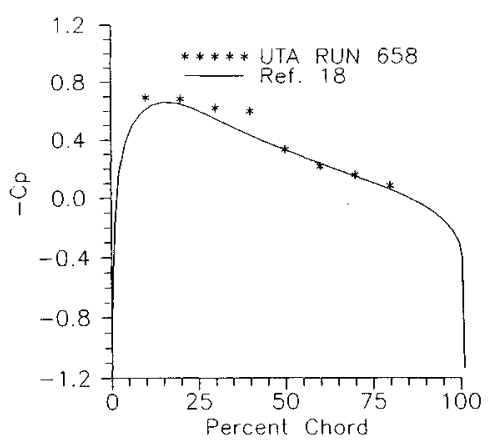

Fig. 6 Vortex-airfoil interaction geometry.

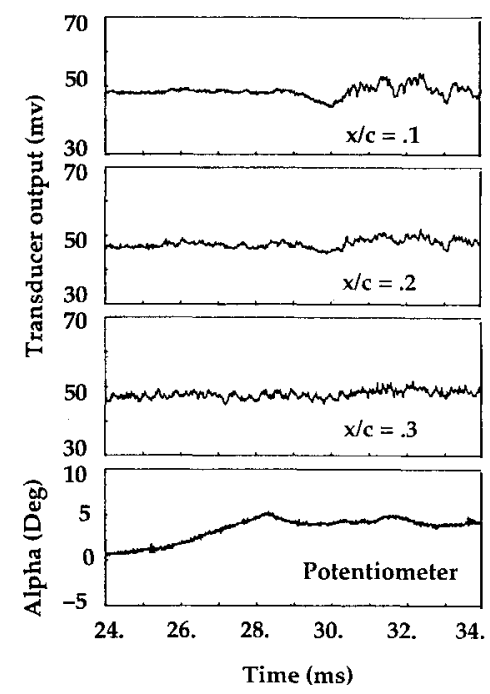

Fig. 7 Time history of pressure signals during the vortex-airfoil interaction: Case C, $M=0.72, R e=3.4 \times 10^{6}, h / c=0$. 
Typical time history plots of the amplified pressure transducer output for $x / c=0.1,0.2$, and 0.3 , along with a superposition of the output signal from the vortex generator pitch angle sensor, is shown in Fig. 7 for case $\mathrm{C}$ at a nominal freestream Mach number of 0.72. A small increase in pressure near the leading edge of the airfoil is observed on the order of $0.7 \mathrm{~ms}$ after initiation of the pitch maneuver, which corresponds roughly to the propagation time for waves traveling with a propagation speed of $u_{\infty}+a$ to reach the downstream airfoil. A drop in pressure is seen to start about $0.7 \mathrm{~ms}$ after termination of the pitch maneuver, which reaches a minimum value about $1.7 \mathrm{~ms}$ after termination of the pitch maneuver. This later time interval roughly corresponds to the convection time for disturbances traveling with the local flow speed $u_{\infty}$ to reach the downstream airfoil. This minimum pressure is then followed by a rapid compression over the leading portion of the airfoil. It is postulated that the starting vortex is shed from the vortex generator at the termination of the pitch maneuver and convects downstream with the local freestream velocity,

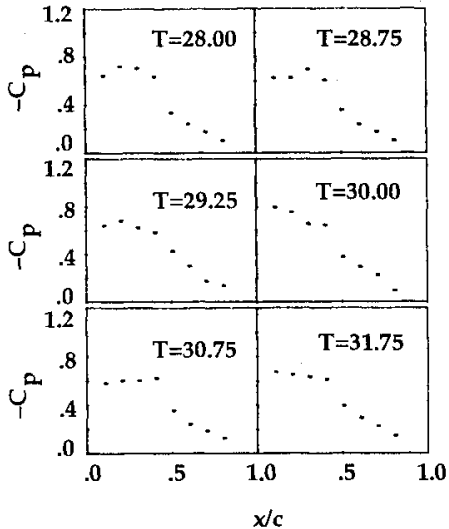

a) Case C: $M=0.72$, $R e=3.4 \times 10^{6}, h / c=0.36$

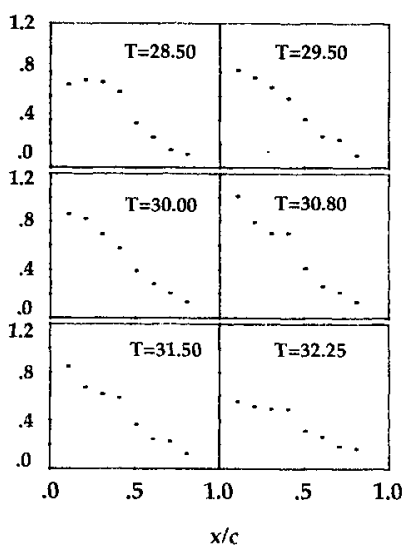

b) Case D: $M=0.72$, $\operatorname{Re}=3.8 \times 10^{6}, h / c=0.48$
Fig. 8 Pressure coefficient distribution during the vortex-airfoil interaction.

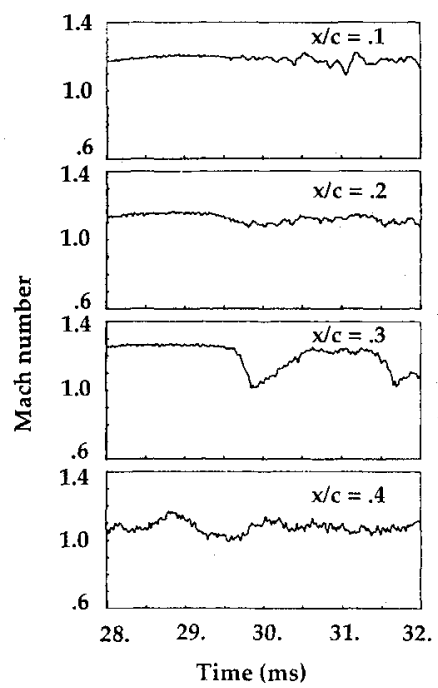

a) Case C: $M=0.77$,

$R e=3.8 \times 10^{6}, h / c=0.36$

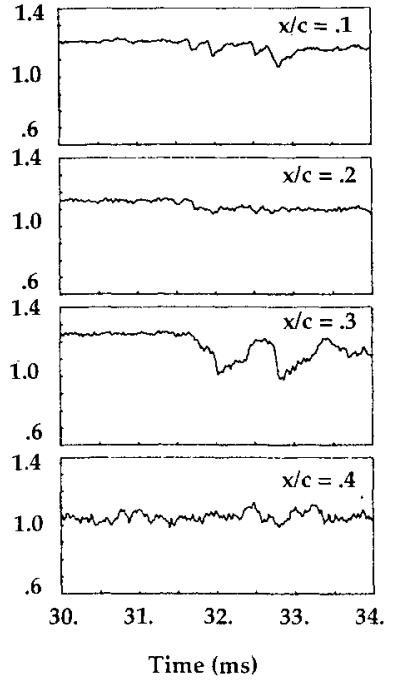

b) Case D: $\quad M=0.78$, $R e=3.8 \times 10^{6}, h / c=0.48$
Fig. 9 Time history of local Mach number distribution during the vortex-airfoil interaction.

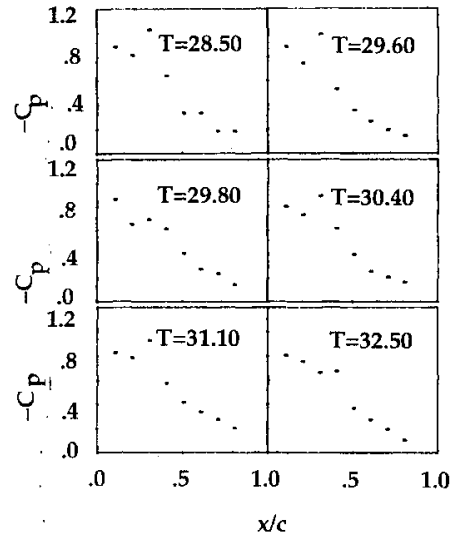

a)

$$
\begin{aligned}
& \text { Case C: } \quad M=0.77 \\
& R e=3.8 \times 10^{6}, h / c=0.36
\end{aligned}
$$

b) Case D: $M=\mathbf{0 . 7 8}$, $\operatorname{Re}=3.8 \times 10^{6}, \bar{h} / c=0.48$
Fig. 10 Pressure coefficient distribution during the vortex-airfoil interaction corresponding to a supercritical flow over the airfoil.

reaching the leading edge of the downstream airfoil $1.7 \mathrm{~ms}$ later. The pressure distribution then oscillates as a result of induced flow oscillations caused by vibration of the vortex generator upon impact with the mechanical stop on the pitch actuation mechanism that is used to set the maximum pitch angle. Such disturbances, however, are believed to follow those induced by the starting vortex and will not be discussed here. Thus, only a small segment of a useful run during which the primary interactions takes place are discussed.

Cases $\mathrm{A}, \mathrm{B}$, and $\mathrm{E}$, representing situations for which the vortex was farther away from the airfoil surface, resulted in only slight modifications to the airfoil pressure distribution and will not be discussed here. On the other hand, the results of interaction studies represented by case $\mathrm{D}$ was similar to case $\mathrm{C}$, and a sensible influence of the vortex on the airfoil pressure distribution was observed. The geometry of the parallel vortex-airfoil interaction represented by cases C and D corresponds to the classic configuration of a clockwise vortex passing under the lower surface of a rotor blade that has been extensively reported in the BVI literature. For all cases, the vortex mostly influenced the leading $30 \%$ of the downstream airfoil, which is in qualitative agreement with the low-speed test results of Refs. 6-7, 10, and 19 and the transonic flow experiments of Ref. 8. This is in contrast to reported results based on numerical simulations of the problem that suggest similar time histories near the leading edge of the airfoil but predict a more uniform chordwise extent of the vortexinduced pressure change over the entire airfoil chord.

The time history pressure traces discussed earlier are presented in the form of the airfoil pressure coefficient distribution in Figs. $8 \mathrm{a}$ and $8 \mathrm{~b}$, for cases $\mathrm{C}$ and $\mathrm{D}$, respectively. These figures do indeed verify that most of the vortex airfoil interaction effects are concentrated near the leading $30 \%$ of the airfoil chord. Figure 8 a shows the instantaneous pressure distribution for a period of $3.75 \mathrm{~ms}$. The initial $C_{p}$ distribution at $T=28 \mathrm{~ms}$ corresponds to the undisturbed pressure distribution approximately $0.4 \mathrm{~ms}$ before the completion of the pitch motion. A gradual decrease in the pressure coefficients at $x / c=0.1,0.2$, and 0.3 is quite evident, whereas downstream of $x / c=0.4$, the pressure distribution is not significantly affected by the passage of the vortex. At $T=30 \mathrm{~ms}$ (about 1.6 ms after the completion of the pitch motion), the airfoil pressure distribution indicates a sensible decrease in the $C_{p}$ near the $x / c=0.1$ station in comparison to its undisturbed value at $T=28 \mathrm{~ms}$. Beyond $T=30.75 \mathrm{~ms}$, the general trend is in the direction of recovering from the unsteady disturbances. However, as indicated earlier, due to the vibration of the vortex generator, the complete recovery is not reached for quite some time. A more pronounced effect on the airfoil pressure distri- 


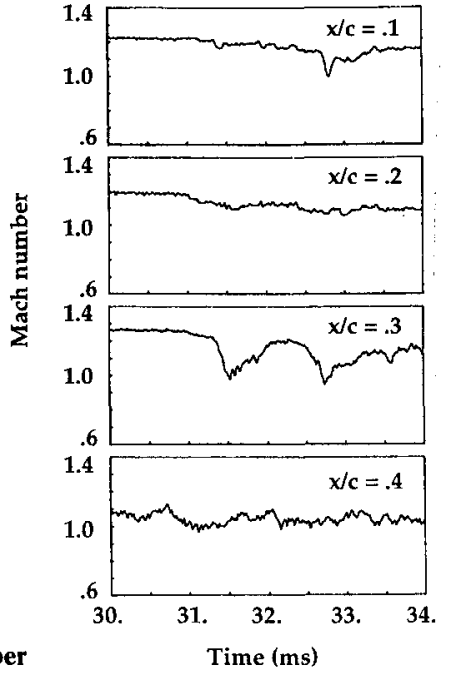

a) Mach number

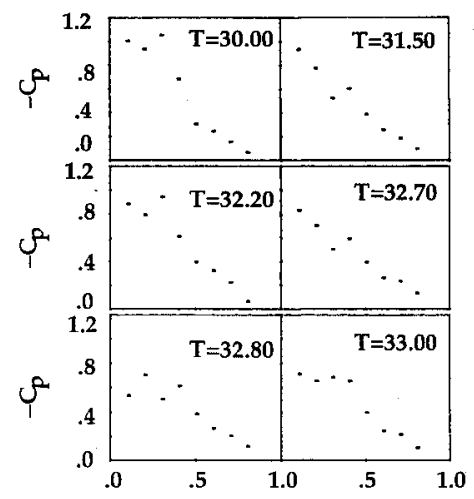

b) Pressure coefficient

$\mathrm{x} / \mathrm{c}$

Fig. 11 Time history of local Mach number and pressure coefficient distribution: $M=0.77, R e=3.8 \times 10^{6}, \Delta \alpha=10 \mathrm{deg}, h / c=0.36$.

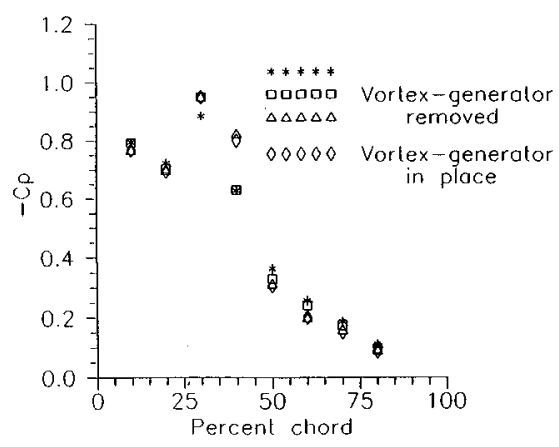

Fig. 12 Comparison of measured upper surface pressure distribution for a nominal Mach number of 0.77 with and without the vortex generator in place.

bution is shown in Fig. 8b for the case in which the downstream airfoil is in position $D$. Again, a progressive decrease in the magnitude of $C_{p}$ values near the leading edge of the airfoil is evident. This trend starts at $T=28.5 \mathrm{~ms}$ (approximately 0.7 ms before the completion of the pitch motion) and continues until $T=30.8 \mathrm{~ms}$ (about $1.7 \mathrm{~ms}$ after the completion of the pitch maneuver), at which time the pressure distribution near the leading edge of the airfoil undergoes compression in the general direction of recovering from the unsteady disturbances.

Experiments with higher freestream Mach numbers resulted in interactions with higher intensity than the subcritical cases discussed earlier. The time history of the local Mach numbers at $x / c=0.1,0.2,0.3$, and 0.4 for the downstream airfoil positions corresponding to cases $C$ and $D$ are presented in
Figs. 9a and 9b, respectively. The previous Mach number traces indicate a strong decrease in the local Mach number at the $30 \%$ chord location just upstream of the steady flow shock wave position, which is an indication of the interaction of the unsteady vortex with the shock wave. Similar to the lower freestream Mach number runs, the supercritical cases resulted in the most pronounced interactions when the vortex passed under the airfoil (instrumented side).

The pressure distribution data for the case in which the downstream airfoil was at position $C$ are illustrated in Fig. 10a. It may be seen that, from $T=29.6 \mathrm{~ms}(0.9 \mathrm{~ms}$ after the completion of the pitch motion) until $T=30.4$, the strong shock wave at $x / c=0.4$ appears to move upstream to a new location at $x / c=0.3$ with a substantial loss in the strength of the shock wave. It may also be seen that this is, to a large degree, a local effect and only the pressures just upstream and downstream of the shock wave are affected by this shock motion. It is also interesting to note that, after about $1.2 \mathrm{~ms}$, the airfoil pressure distribution is essentially the same as its undisturbed value. A similar behavior may be observed in Fig. $10 \mathrm{~b}$ for case $\mathrm{D}$.

The forward propagation of the shock wave during the interaction was found to be more pronounced if the vortex generator was pitched from a preset negative angle to a positive angle of attack. Such a pitch motion will result in generation of a stronger vortex without separating the flow over the vortex-generator airfoil. This maneuver should shed a vortex with a nondimensional strength $\bar{\Gamma}$ of 0.5 for a pitch excursion from -5 to $+5 \mathrm{deg}$. An example of such a case is presented in Fig. 11. Figure 11a illustrates the time history plots of the local Mach numbers for $x / c=0.1-0.4$. Although the picture is complicated by the presence of a weak shock wave at $x /$ $c=0.2$, it is apparent that a sharp decrease in the local Mach number at $x / c=0.3$ occurs approximately $0.7 \mathrm{~ms}$ before the disturbances are felt at $x / c=0.1$. The pressure coefficient distribution for this case is illustrated in Fig. 11b for a period of $3 \mathrm{~ms}$. At $T=30 \mathrm{~ms}$ the undisturbed pressure distribution indicates a very strong shock wave at $x / c=0.4$. At $T=31.5$ $\mathrm{ms}$, the shock wave has moved upstream to $x / c=0.3$ with a substantial loss in its strength, whereas the aft portion of the airfoil does not seem to be affected by the interaction. At $T=32.2 \mathrm{~ms}$, the distribution is very similar to the undisturbed values before a second disturbance results in another upstream shock movement, leading to compression of the leading portion of the airfoil. This leads to what appears to be a pattern of unsteady flow separation and reattachment near the airfoil leading edge ( $T=33 \mathrm{~ms})$.

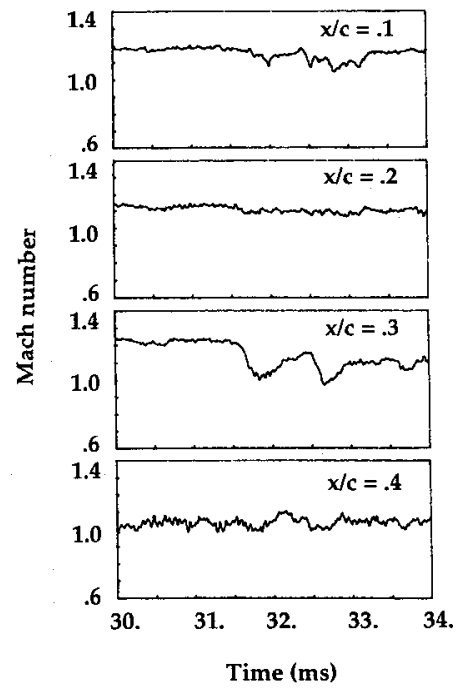

a) $R e=3.8 \times 10^{6}$

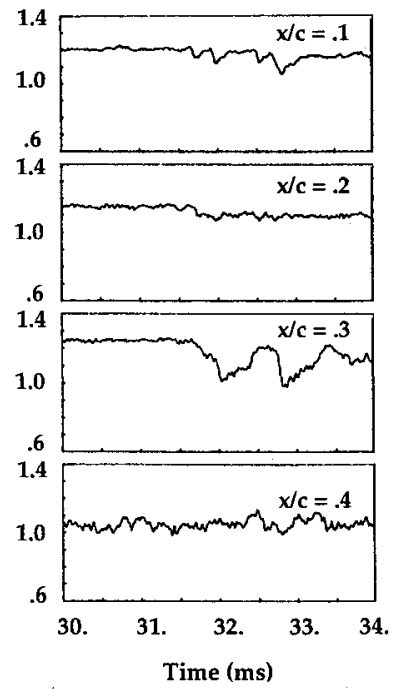

b) $R e=5.4 \times 10^{6}$
Fig. 13 Illustration of Reynolds number effect: $M=0.77, h / c=0.48$. 
As mentioned earlier, the airfoil pressure distribution for Mach numbers representing supercritical flows over the airfoil showed poor correlation with those of a NACA 0012 airfoil section. Figure 12 shows the comparison of the airfoil pressure distribution in the absence of the vortex generator with the data when the vortex generator was in place (prior to the interaction). Such trends suggest that the results are quite repeatable, and the phenomena observed should give an accurate account of the unsteady vortex-airfoil interaction.

The variation of flow Reynolds number, as shown in Fig. 13, does not appear to have a significant effect on the downstream airfoil. Figure 13 illustrates the Mach number traces for $M=0.77$ at $x / c=0.1,0.2,0.3$, and 0.4 and chord Reynolds numbers of $3.8 \times 10^{6}$ and $5.4 \times 10^{6}$. Except for minor differences in time correlation of the large amplitude pressure variations at $x / c=0.3$, the traces are essentially identical.

\section{Conclusions}

Analysis of the airfoil pressure distribution revealed several propagation mechanisms. First, relatively weak disturbances were observed a short time after the initiation of the vortex generator pitch motion. The propagation speed for these disturbances was approximately representative of waves traveling with a speed of $u_{\infty}+a$. Second, continuous but more pronounced disturbances were noted some time after the completion of the pitch maneuver with propagation speed of approximately $u_{\infty}+a$. These disturbances were terminated by rapid but continuous pulses representative of particles leaving the vortex generator and convecting downstream with the local freestream velocity. The results of the present investigation indicated that the unsteady interaction of a vortex with a two-dimensional airfoil can have a significant effect on the pressure distribution of the interacting airfoil. The experimental results are qualitatively similar to those obtained from computational analysis of the parallel BVI problem for subcritical and critical Mach numbers with one notable exception: experimental results indicate that the interaction is more pronounced over the leading $40 \%$ of the airfoil chord, whereas the numerical simulations suggest a more uniform chordwise extent of the interaction. Experimental data for freestream Mach numbers representing supercritical flow over the airfoil indicate that a strong interaction between the vortex and the shock wave can lead to shock wave oscillation and local flow separation. For this condition, an increase in the strength of the vortex was seen to produce a forward propagation of the shock wave (type $\mathrm{C}$ shock motion). In general, the effect of Reynolds number variation over the range tested appears to be of second order.

\section{Acknowledgments}

The parallel blade-vortex interaction experiments came from a study conducted while the first author was a visiting assistant professor at the University of Texas at Arlington. This work was supported by the U.S. Army Research Office (ARO Grant DAAG29-84-K131); Dr. Thomas Doligalskey was Contract Monitor.

\section{References}

${ }^{1}$ George, A. R., "Helicopter Noise: State-of-the-Art," Journal of Aircraft, Vol. 15, No. 11, 1978, pp. 707-715.

${ }^{2}$ Ham, N. D., "Some Conclusions from an Investigation of BladeVortex Interaction," Journal of the American Helicopter Society, Vol. 20, No. 4, 1976, pp. 26-31.

${ }^{3}$ Schlinker, R. H., and Amiet, R. K., "Rotor-Vortex Interaction Noise," AIAA 8th Aeroacoustics Conference, AIAA Paper 83-0720, Atlanta, GA, April 1983.

${ }^{4}$ Ahmadi, A. R., "An Experimental Investigation of Blade-Vortex Interaction at Normal Incidence," Workshop on Blade-Vortex Interaction, NASA Ames Research Center, Oct. 1984.

5Seath, D. D., and Wilson, D. R., "Vortex-Airfoil Interaction Tests," AIAA 24th Aerospace Sciences Meeting, AIAA Paper 860354, Reno, NV, Jan. 1986.

${ }^{6}$ Seath, D. D., Kim, J. M., and Wilson, D. R., " An Investigation of the Parallel Blade-Vortex Interaction in a Low-Speed Wind Tunnel," Journal of Aircraft, Vol. 26, No. 4, 1989, pp. 328-333.

${ }^{7}$ Straus, J., Renzoni, P., and Mayle, R. E., "Airfoil Pressure Measurements During a Blade-Vortex Interaction and a Comparison with Theory," AIAA 26th Aerospace Sciences Meeting, AIAA Paper 880669, Reno, NV, Jan. 1988.

${ }^{8}$ Caradonna, F. X., Laub, G. H., and Tung, C., “An Experimental Investigation of the Parallel Blade-Vortex Interaction," 10th European Rotocraft Forum, Paper 4, The Hague, The Netherlands, Aug. 1984.

${ }^{9}$ Booth, E. R., "Experimental Observations of Two-Dimensional Blade-Vortex Interaction," AIAA Journal, Vol. 28, No. 8, 1990, pp. 1353-1359.

${ }^{10}$ Booth, E. R., and Yu, J. C., "Two-Dimensional Blade-Vortex Flow Visualization Investigation," AIAA Journal, Vol. 24, No. 9, 1986, pp. 1468-1473.

11Poling, D. R., Wilder, D. P., and Telionis, D. P., "Two-Dimensional Interaction of Vortices with a Blade," AIAA 26th Aerospace Sciences Meeting, AIAA Paper 88-0044, Reno, NV, Jan., 1988.

${ }^{12}$ Meier, G. E. A., and Timm, R., "Unsteady Vortex Airfoil Interaction," AGARD CP-386, May 1985.

${ }^{13}$ Mandella, M., and Bershader, D., "Qualitative Study of the Compressible Vortex: Generation, Structure and Interaction with Airfoils," AIAA 25th Aerospace Sciences Meeting, AIAA Paper 870328, Reno, NV, Jan. 1987.

${ }^{14}$ Srinivasan, G. R., McCroskey, W. J., and Kutler, P., “'Numerical Simulation of a Vortex with Stationary Airfoil in Transonic Flow," AIAA 22nd Aerospace Sciences Meeting, AIAA Paper 840254, Reno, NV, Jan. 1984.

${ }^{15}$ Srinivasan, G. R., and McCroskey, W. J., "Unsteady Interaction of a Rotor with a Vortex," AIAA 20th Fluid Dynamics, Plasma Dynamics, and Lasers Conference, AIAA Paper 89-1848, Buffalo, NY, June 1989.

${ }^{16}$ Wilson, D. R., and Chou, S. Y., "Development of the UTA High Reynolds Number Transonic Wind Tunnel," AIAA 23rd Aerospace Sciences Meeting, AIAA Paper 85-0315, Reno, NV, Jan. 1985.

${ }^{17}$ Kalkhoran, I. M., "An Experimental Investigation of the Perpendicular Vortex-Airfoil Interaction at Transonic Speeds," Ph.D. Dissertation, Aerospace Engineering Department, Univ. of Texas at Arlington, Arlington, TX, Dec. 1987.

${ }^{18}$ Lock, R. C., "Test Cases for Numerical Methods in Two-Dimensional Transonic Flows," AGARD Rept. 575, 1970.

${ }^{19} \mathrm{Yu}$, J. C., "Flow Field Visualization of Two-Dimensional BladeVortex Interactions," Workshop on Blade-Vortex Interaction, NASA Ames Research Center, Oct. 1984. 